\title{
Hechos estilizados del ciclo económico de Ecuador: 1990-2009
}

\section{Mercy Orellana ${ }^{\star}$}

\begin{abstract}
Resumen
Este trabajo presenta un conjunto de "hechos estilizados" del ciclo económico en Ecuador. Para describir las propiedades de las fluctuaciones económicas se siguió la metodología de Kydland y Prescott (1990). Se documentan las volatilidades del componente cíclico de las diferentes variables macroeconómicas así como los co-movimientos con el componente cíclico del PIB real. Con el propósito de analizar posibles cambios en las propiedades cíclicas del ciclo económico ecuatoriano, el período de análisis se divide en dos sub-períodos, 1990-1999 y 2000-2009, esto debido a la adopción de la dolarización oficial a inicios de 2000. Los ciclos reales de Ecuador presentan algunas interesantes particularidades. El comercio representa una parte importante de la actividad económica; las exportaciones y los términos de intercambio tienden a liderar el ciclo. Por otro lado, la política cambiara y monetaria juegan un rol significativo en las fluctuaciones de corto plazo.
\end{abstract}

\section{Palabras clave}

Ciclo económico, dolarización, intercambio comercial, métodos de análisis económico, recesión.

\begin{abstract}
This paper presents a set of "stylized facts" of the business cycle in Ecuador. To describe the properties of economic fluctuations I followed the Kydland and Prescott methodology (1990). It documents the volatility of the cyclical component of macroeconomic variables and the co-movements with the cyclical component of real GDP. In order to analyze possible changes in the cyclical properties of the Ecuadorian economic cycle, the period of analysis is divided into two sub-periods, 1990-1999 and 2000-2009, this due to the adoption of official dollarization in early 2000. Ecuador real cycles have some interesting peculiarities. Trade is an important part of economic activity; exports and the terms of trade tend to lead the cycle. On the other hand, change and monetary policy play a significant role in short-term fluctuations.
\end{abstract}

\section{Key words}

Business cycle, dollarization, trade, economic analysismethods, recession.

Forma sugerida de citar: Orellana, Mercy. 2011. "Hechos estilizados del ciclo económico de Ecuador: 1990-2009”. Universitas 15. Julio/Diciembre. Pp. 53-84.

\footnotetext{
* Profesora e investigadora de la Universidad Estatal de Cuenca. E-mail: mqorella@uc.cl
} 


\section{Introducción}

Las fluctuaciones recurrentes en la actividad económica son comunes tanto en economías desarrolladas como en desarrollo; así, diferentes teorías han intentado explicar este fenómeno con relativo éxito. La teoría moderna de ciclo económico establece dos objetivos básicos en la investigación del ciclo económico: la caracterización completa y sistemática de los fenómenos cíclicos en la forma de "hechos estilizados", ${ }^{2}$ y la construcción de modelos completos de equilibrio general que puedan ser evaluados cualitativa y cuantitativamente en términos de su capacidad para reproducir estos hechos.

Este trabajo se enmarca en la investigación del primero de estos objetivos. El propósito principal desde una perspectiva "ateórica", pretende identificar la mayoría de hechos estilizados relacionados con los ciclos económicos en Ecuador. Éstos constituyen un benchmark contra el cual posibles explicaciones de fuentes y mecanismos de las fluctuaciones cíclicas en Ecuador pueden ser confrontados, además de permitir discriminar entre diferentes modelos alternativos.

Para estudiar los hechos estilizados de los ciclos económicos ecuatorianos se sigue la metodología de Kydlandy Prescott (1990). Se estudian los patrones cíclicos del PIB, así como también de otras variables clave. Es decir, se desagrega el PIB en elementos por el lado de la demanda, insumos productivos, variables nominales y variables que se relacionan con una economía abierta. La recopilación de hechos estilizados del ciclo económico es importante por dos razones. Primero, nos da un resumen de los co-movimientos existentes entre los agregados en la economía, además que permite tener una aproximación de la magnitud de las fluctuaciones de las variables económicas y, de esta manera, nos admite el escoger indicadores líderes para la actividad económica. Segundo, provee un set de "regularidades" las cuales pueden ser usadas como un benchmark para examinar la validez de los modelos teóricos.

El primer objetivo de este estudio es tener una valoración sobre los co-movimientos, la persistencia y la volatilidad de cada una de las variables macroeconó-

2 Por hechos estilizados se alude a regularidades generales en las propiedades estadísticas de las series económicas. 
micas relevantes, para datos trimestrales desde 1990 a 2009. La caracterización de los co-movimientos es importante para la discusión de los hechos estilizados del ciclo económico. Este análisis se complementa mediante la discusión de algunas de las implicaciones teóricas de los fenómenos encontrados. A fin de determinar posibles particularidades, los resultados se comparan con estudios similares a éste para economías de Latinoamérica y para Estados Unidos.

Finalmente, el período de estudio también se divide en dos subperíodos, 1990-1999 y 2000-2009. Dicha periodización se debe a que en el 2000, Ecuador adoptó el dólar como moneda de circulación legal. La mayoría de trabajos de ciclo económico internacionales, establecen que el régimen de tipo de cambio es de principal importancia para explicar las fluctuaciones macroeconómicas. Además, existe abundante evidencia que por lo menos una variable real (el tipo de cambio real), cambia significativamente bajo tipo de cambio fijo $^{3}$ y flexible.

Dado que el tipo de cambio real es visto como un precio relativo del producto interno, la diferencia en la volatilidad de este precio relativo sugiere que puede provocar diferencias en el comportamiento del producto, consumo inversión, oferta laboral, y otras variables claves (Baxter, 1991). Así, el último objetivo es determinar si el comportamiento estadístico de los agregados macroeconómicos de Ecuador ha cambiado sustancialmente, antes y después de la dolarización. Al no existir trabajos sobre este tema para Ecuador, la principal contribución de este estudio es presentar en detalle los hechos estilizados que caracterizan el ciclo económico ecuatoriano.

El resto del documento se organiza de la siguiente manera. En la Sección II se presenta una breve revisión histórica de las recesiones en Ecuador desde 1980, usando datos anuales. Se discute la literatura teórica y empírica sobre los ciclos económicos en la sección III. En la siguiente sección se evalúa el método usado para extraer el componente cíclico de cada serie y los momentos estadísticos de los mismos. En la Sección V se analiza el comportamiento de las variables macroeconómicas en el período 1990-2009 para documentar las características principales del ciclo económico en Ecuador. Finalmente, las conclusiones son propuestas en la Sección VI. 


\section{Revisión histórica de las recesiones en la economía Ecuatoriana desde 1980}

Durante los últimos 30 años el PIB ecuatoriano ha crecido a una tasa promedio de 2,6\%, también ha presentado amplias fluctuaciones año a año, desde un gran $10,9 \%$ en 1988 a solo un $-6,51 \%$ en 1999 . En contraste, en el mismo período el crecimiento de los USA fue de $2,6 \%$ y fluctuó en un rango cercano entre $-1 \%$ a 4\%. En lo últimos 10 años después de la adopción de la dolarización, Ecuador ha evidenciado una notable reducción en la inflación, desde una tasa de $99 \%$ en el 2000, a una tasa menor al 3\% en 2007, lo cual implica que la contribución de tanto las fluctuaciones nominales como reales han jugado un rol importante durante este período.

Gráfico 1. Tasa de variación anual del PIB real Ecuador: 1980-2009

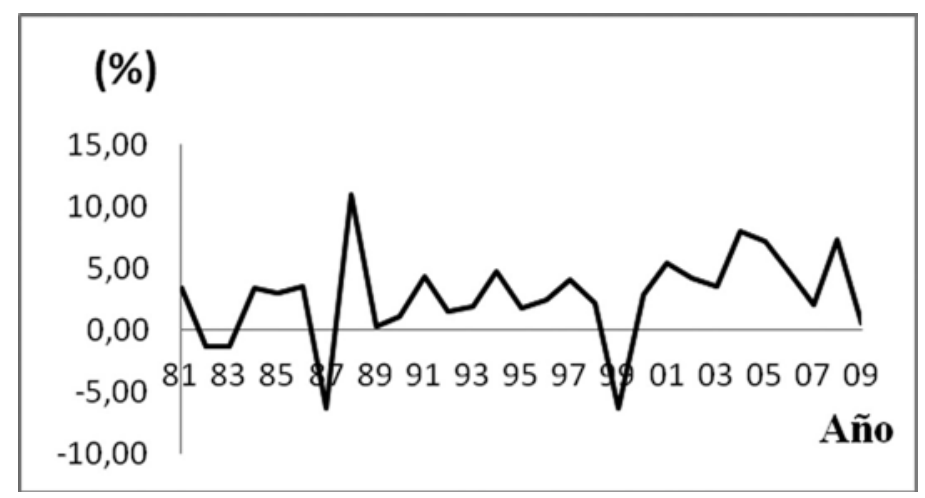

Fuente: Banco Central del Ecuador

\section{Recesiones de los años ochenta}

Los años ochenta se inician con cambios importantes tanto de orden político como económico, los que determinaron una nueva estrategia en la aplicación de los programas de gobierno. Estos son en primer término, el retorno en 1979 al régimen democrático $\mathrm{y}$, segundo, el agravamiento de los desequilibrios económicos que venían acumulándose desde la década anterior (Fernández, 1994). 


\section{Crisis 1982-1983}

La crisis ecuatoriana de estos años se desarrolla en un medio internacional desfavorable, moratoria de la deuda externa mexicana, altas tasas de interés en el mercado internacional, la contracción del comercio internacional y un deterioro de los términos de intercambio que provocaron una disminución en las exportaciones de 6,92\%. En el trascurso de un año, la inflación pasó de $14,45 \%$ a un $63.28 \%$. A este incremento en los niveles inflacionarios se sumó la fuerte caída de casi un $63 \%$ de las reservas internacionales, que acompañadas de un déficit de cuenta corriente, demostraban la vulnerabilidad de la economía para enfrentar shocks externos. Para ajustar los desequilibrios el Gobierno se propuso disminuir el déficit fiscal que para 1982 llegaba a su cifra más alta: 4,2\% del PIB, acompañada de una política cambiaria más flexible mediante un sistema de cambio de mini devaluaciones; para 1983 el sucre se había devaluado en un 63,94\%, convirtiéndose ésta en una de las principales políticas estabilizadoras del país.

\section{Crisis de 1987}

Nuevamente en el año 1986 un shock negativo de precios del petróleo golpeó la economía ecuatoriana, provocando una disminución de $24 \%$ en los términos de intercambio, la crisis del sector externo se hizo evidente para 1987, lo que provocó una disminución de las reservas internacionales de 138,54\% (saldo negativo). Las exportaciones se contrajeron en un $28,45 \%$. La caída del producto fue una de las más fuertes que ha tenido el país en estas tres décadas $-6,47 \%$. La inflación pasó de un $29 \%$ a un $56 \%$ a finales de 1987 . Una vez más las políticas de ajustes fueron: devaluación cambiaria, control del gasto público y la suspensión de pagos de deuda, lo que produjo una fuerte restricción de capitales externos. Así, las crisis sufridas en la década de los ochenta muestran elementos típicos comunes que corresponden a factores externos para las recesiones ecuatorianas: un shock negativo de términos de intercambio.

\section{Recesiones años noventa}

En los noventa comenzó a prevalecer una política de apertura externa y liberalización. Indicadores importantes como la tasa de interés y el tipo de cambio 
se determinaron de acuerdo a las condiciones del mercado y entre los objetivos más importantes se encuentran: la reducción de la inflación, el fortalecimiento de la posición fiscal y una sustancial reforma al sector público. Para finales de 1996, las reservas internacionales habían aumentado notablemente en un 66\%. La inflación registró una notable baja de su nivel al pasar de 54\% en 1992 a $24 \%$ a finales de 1996. A su vez, mejoró la situación de las finanzas públicas, se registró un superávit fiscal de 3,3\% en relación al PIB. El aumento de las exportaciones no petroleras permitió mantener una balanza comercial positiva.

No obstante, en medio de la relativa estabilidad económica, en 1998 se registran una serie de acontecimientos que tuvieron un fuerte golpe para el país.El impacto del fenómeno El Niño provocó estragos en la infraestructura y el sector agrícola, la baja del precio del petróleo provocó un deterioro de los términos de intercambio de casi un $14 \%$, la crisis financiera internacional produjo problemas de financiamiento externo. Estos hechos, conjuntamente con la crisis política, provocaron salida de capitales, una contracción del $20 \%$ en las exportaciones y una fuerte presión cambiaria debido a factores especulativos. La banda de flotación cambiaria fue recalibrada en dos ocasiones y la inflación rebrotó a consecuencia de los efectos del desastre climático sobre la producción agrícola y el transporte llegando a una tasa del $45 \%$.

\section{Crisis de 1999}

A partir de una profunda crisis de los sistemas financiero y cambiario, la economía ecuatoriana se contrajo alrededor de 7,3\%, para finales de 1999, la inflación subió hasta un $60 \%$, la emisión alrededor de un $150 \%$ y la depreciación aumentó más de un 190\%. La demanda interna disminuyó en un $10 \%$, lo que produjo un aumento del desempleo (16\%) y el subempleo (57\%). El producto percápita de los ecuatorianos en 1999 fue menor al que obtenían en 1980 en cerca de 100 dólares, el déficit fiscal aumentó a un 4,2\% con respecto al PIB. En una situación de déficit fiscal y de alta carga del servicio de la deuda en 1999, Ecuador dejó de pagar los bonos Brady, ${ }^{4}$ que luego se extendió a los eurobonos

4 Bonos Brady: Obligación emitida por gobiernos soberanos de mercados emergentes en sustitución de deuda bancaria como consecuencia de una renegociación entre el deudor y sus acreedores. 
y a la deuda privada. Para evitar una depreciación aún mayor, proteger el sistema financiero y contener la inflación, en marzo se decretó el congelamiento de una parte de los depósitos financieros. Esto frenó la demanda y restringió las posibilidades de financiamiento de las empresas. La cartera vencida de la banca privada subió de 9,2\% a fines de 1998 a 51,8\% en noviembre de 1999. El debilitamiento de la actividad impidió que la inflación se descontrolara pese a la acelerada depreciación del sucre y al fuerte aumento de la emisión monetaria.

En contraste, un mejoramiento de los precios del petróleo y de los términos de intercambio ayudó a un aumento de las exportaciones petroleras, de esta manera, las exportaciones a final del período habían disminuido en un 3\%, sin embargo, las importaciones disminuyeron un 35\%, y esto generó un importante superávit de la balanza comercial, lo cual provocó un excedente en la cuenta corriente de $6 \%$ del PIB. Se dificultó el acceso al crédito externo y la inversión extranjera directa presentó un leve retroceso. Por tanto, se produjo una reducción de las reservas internacionales de un 40\% entre 1998 y 1999. El público empezó a demandar en forma creciente dólares, presionando a la tasa de cambio. Es así como en 1998 el tipo de cambio de 6.521,00 pasa a 18.287,00 sucres por dólar para 1999. Empezó a ser evidente que las tasas de interés en los depósitos a plazo tendían a ser negativas en términos reales, a pesar de la política del Banco Central del Ecuador de elevarlas nominalmente.

En reacción a los graves trastornos financieros y cambiarios del año anterior, en enero de 2000 las autoridades decidieron sustituir el sucre por el dólar estadounidense. El proyecto sobrevivió incluso a la crisis política que culminó con la deposición del Presidente de la República, y al cabo de una transición de seis meses el dólar se transformó en la única moneda de curso legal en Ecuador. Sin embargo, la inflación se mantuvo muy elevada, con lo que a fines de diciembre de 2000 se sitúo en el rango del $90 \%$, luego de este período la inflación empezó a presentar una tendencia a la baja. Se logró revertir la caída del producto el mismo que creció en un $2 \%$. A pesar de un repunte de las importaciones, la cuenta corriente registró un superávit, causado por la marcada mejoría del precio del petróleo. 


\section{Año 2000, dolarización y recesiones}

Durante estos nueve años de dolarización el objetivo de reducir la inflación a un dígito se ha logrado cumplir, es así que en el 2009, la tasa de inflación fue de $5,9 \%$. Otra variable clave que se redujo notablemente fue la tasa de interés activa de corto plazo que cayó a un 9,19\%, en comparación a la de 1999 que llegaba a un $69 \%$. De hecho, la apreciación del tipo de cambio real después de la dolarización es uno de los efectos más importantes de la reforma, y con frecuencia uno de los menos comprendidos. En la ausencia de aumentos de la productividad laboral, la única alternativa para un país de aumentar su competitividad en el exterior es por la depreciación de su tipo de cambio (Bradbury y Vernengo, 2008). Sin embargo, Ecuador adoptó la dolarización en un período en que el dólar ha perdido en promedio un $4 \%{ }^{5}$ de su valor frente a otras monedas, aumentando las exportaciones ecuatorianas en el proceso. Cabe recalcar que hasta el 2008, no existieron shocks internacionales fuertes que golpearan a la economía, el crecimiento del producto alcanzó en el 2008 un 7\%, con los precios del petróleo que se mantenían altos.

En el año 2009 se presentaron una serie de problemas tanto internacionales como internos, que reflejaron nuevamente la vulnerabilidad de la economía ecuatoriana. La crisis financiera mundial y el deterioro de los términos de intercambio, afectaron negativamente al crecimiento del PIB que alcanzó un $0,36 \%$. Nuevamente la caída de las exportaciones debido a un deterioro de los términos de intercambio, señala el carácter de indicador líder que el petróleo tiene en las recesiones ecuatorianas.

\section{Literatura relevante}

El conocimiento de los "hechos estilizados" juega un rol importante en la macroeconomía, en el sentido que éstos describen cuáles son los hechos que deben ser explicados por los modelos teóricos. La noción de hechos estiliza-

5 Depreciación promedio del tipo de cambio nominal durante los últimos 10 años: frente al Euro 4\%, nuevo sol peruano $2 \%$ y una apreciación de $0,9 \%$ con respecto al peso colombiano. La elección de los países o regiones se debe a que son los principales socios comerciales de Ecuador. 
dos tiene sus raíces en el trabajo de Burns y Mitchell (1946) cuya idea básica fue proveer características de los datos de una forma ateórica. Esta idea fue impugnada por Koopmans (1947) quien argumentó que la teoría económica debe entrar de dos maneras. En primer lugar, la teoría económica sirve como un mecanismo para diferenciar los hechos importantes de los de poca importancia. En segundo lugar, las descripciones de los fenómenos económicos no pueden hacerse sin especificar los modelos econométricos estructurales basados en esquemas de oferta y demanda.

Sin embargo, en el trabajo de Kydland y Prescott (1990) se acepta la primera crítica pero se rechaza la segunda. Así, reportar las características de los ciclos económicos se ha convertido en un ejercicio cotidiano en la macroeconomía a partir de este trabajo. Estos autores destacan la importancia de contar con un conjunto mínimo de hechos estilizados sobre los ciclos económicos que permitan validar los modelos que se utilizan para hacer análisis macroeconómico. En su trabajo, Kydland y Prescott siguiendo a Lucas (1977) definen a los ciclos económicos como desviaciones repetitivas del producto desde su tendencia y el comportamiento relativo con otros agregados monetarios. Entre sus principales resultados señalan el comportamiento procíclico de los precios en Estados Unidos, además no encuentran evidencia favorable a la hipótesis monetarista de que algún agregado monetario como la emisión o M1 lideren o conduzcan al ciclo económico. No obstante, encuentran que la diferencia entre M2-M1, es decir el canal del crédito, sería un buen indicador líder del ciclo, inclusive mejor que M2. Para obtener el componente de tendencia y ciclo de las series $\mathrm{y}$, posteriormente, establecer las relaciones de movimiento entre las principales variables macroeconómicas y el producto a lo largo del ciclo económico, utilizan la metodología desarrollada por Hodrick y Prescott (1980). Este filtro (H-P) captura el movimiento secular o tendencial de una determinada variable, admitiendo la posibilidad de que ocurran cambios en la tendencia.

Estudios posteriores han aplicado esta metodología "ateórica" para documentar la evidencia del ciclo económico en distintos países. Igualmente, Backus y Kehoe (1992) aplican la misma metodología para 10 países de la OECD (Organización para la Cooperación y el Desarrollo Económico), ellos dividen los datos en pre y posguerra y entre sus principales resultados encuentran una disminución en la volatilidad del producto en los datos de posguerra, además 
encuentran que el componente cíclico del consumo y la inversión son procíclicos al producto, sin embargo la desviación estándar de la primera es igual que la del producto, pero para la inversión es tres veces más volátil. Reportan dos cambios significativos en el comportamiento cíclico de los precios, en el período antes de la guerra el producto y el nivel de precios estaban positivamente correlacionados, más allá de que en los datos de posguerra los precios tienen un comportamiento contra-cíclico. Encuentran evidencia de disminución en las correlaciones entre el dinero y el producto en el período de posguerra. Además encuentran un aumento significativo en la persistencia del movimiento de los precios en la mayoría de los países considerados.

Fisher, et al.(1994) utilizan el filtro Hodrick y Prescott para reportar los principales hechos del ciclo económico australiano entre sus principales resultados está el comportamiento procíclico de la inversión y el consumo; la volatilidad del primero es mayor que el producto pero la volatilidad del consumo es menor que la volatilidad del PIB. El consumo del gobierno parece no correlacionado con el ciclo. Las exportaciones son procíclicas y contemporáneas con el ciclo. Al igual que los resultados de Hodrick y Prescott para los EE.UU., encuentran que el nivel de precios es contra-cíclico y que la base monetaria rezaga al ciclo por cerca de dos trimestres, lo que sugeriría que los shocks monetarios no son una fuente significante de las fluctuaciones económicas en Australia.

Sin embargo, ya sea por la ausencia de datos o estabilidad económica en los países de Latinoamérica la mayoría han dejado de lado esta línea de investigación. Entre los primeros trabajos realizados para estas economías está el trabajo de Kydland y Zarazaga (1997), aplicando la misma metodología de Kydland y Prescott (1990), documentan las propiedades del ciclo económico en Argentina. El principal problema que enfrentan es la disponibilidad de datos y usan dos versiones no consolidadas de las cuentas nacionales. Por este motivo los hechos estilizados del ciclo económico no son robustos en las distintas bases de datos. Entre las principales conclusiones que reportan está la gran volatilidad del producto en comparación con EE.UU., además encuentran que los resultados obtenidos no son favorables a la hipótesis de que los factores nominales desempeñan el papel más importante en las fluctuaciones económicas. En particular, el nivel de precios es contra-cíclo, al igual que los agregados monetarios. Finalmente, los autores concluyen que los factores nominales no parecen ser capaces 
de dar cuenta de cualquier fracción importante de los ciclos económicos de los países de América Latina en particular de Argentina.

Agenor, et al. (1998) en su estudio incluyen a Chile, Colombia, México y Uruguay, además de siete países de ingreso medio, para investigar la existencia de un set de hechos estilizados de los ciclos económicos en estos países. El documento se concentra en los ciclos de un índice de producción industrial, utilizan dos procedimientos para obtener el componente cíclico: el filtro de Hodrick-Prescott (H-P) y Baxter-King (B-K). Entre los principales resultados encuentran que la volatilidad del producto medida por la desviación estándar varía sustancialmente entre los países en desarrollo, además en promedio la volatilidad es mucho mayor que los países industrializados. Hallan evidencia favorable a la hipótesis que los salarios reales son procíclicos. Encuentran que no hay una relación consistente entre el nivel de precios y la inflación con el producto. Reportan que la correlación entre el dinero y el producto es contemporánea positiva pero no muy fuerte. Sus resultados sugieren la importancia de los shocks de oferta en la conducción del ciclo económico en los países en desarrollo.

A pesar de esto, Belaisch y Soto (1998), encuentran que el "mito monetario" encontrado por Kydland y Prescott para la economía de los Estados Unidos está vivo en Chile, entre sus principales resultados se tiene la excesiva sensibilidad del consumo a las fluctuaciones del producto; la volatilidad de la inversión es aproximadamente cuatro veces mayor que la del producto. Encuentran correlación contemporánea de las exportaciones con el producto señalando que las exportaciones podrían jugar un rol importante en la determinación de las fluctuaciones de corto plazo en la economía chilena. Reportan un movimiento contra-cíclico de los precios y procíclicos de los salarios reales. Mas las fluctuaciones en M1 son fuertemente procíclicas y lideran el ciclo hasta por dos trimestres.

Retrepo y Reyes (2000) repiten el ejercicio realizado por Kydland y Prescott para estudiar los ciclos de la economía colombiana, encuentran una excesiva volatilidad de la inversión y un comportamiento acíclico de las exportaciones. Reportan que los insumos productivos presentan un comportamiento procíclico con una volatilidad menor que la del PIB. Además que reportan conclusiones diferentes a las de Agenor et al. (1998), los precios y las distintas medidas de 
dinero son procíclicas y sus correlaciones son contemporáneas. Concluyen que las fluctuaciones cíclicas de la economía colombiana estarían siendo explicadas principalmente por choques de demanda.

Entre los principales trabajos que relacionan el ciclo económico con el tipo de cambio está el documento de Baxter y Stockman (1988), quienes investigan si las propiedades estadísticas de los ciclos económicos de 43 países dependen de la elección del tipo de cambio, para eso comparan el comportamiento de las principales series macroeconómicas bajo el sistema de tipo de cambio fijo Bretton Woods con el sistema de tipo de cambio flotante que prevaleció desde 1973, utilizan tres métodos alternativos para descomponer la serie en ciclo y tendencia.

La conclusión de este estudio es que algunas series como las de comercio exterior y la producción industrial, se han vuelto más volátiles bajo tipo de cambio flexible, aunque la magnitud del aumento es muy pequeña, sin embargo, hay poca evidencia que esos cambios se deban a la elección del tipo de cambio.

Baxter (1991) realiza la misma investigación anterior pero solo para la economía de EE.UU., sus principales resultados son: bajo el período de tipo de cambio flexible, la volatilidad de la inversión privada fue mayor, pero el gasto en equipos de planta fue menor. La volatilidad de las importaciones fue sustancialmente más alta en el período de tipo de cabio flexible, aunque la volatilidad de las exportaciones disminuyó. Además hay evidencia que la volatilidad del consumo incrementó en el período pos 1973. Cabe destacar que sus resultados son sensibles al método utilizado para obtener la tendencia de los datos.

Ahmed, S. (2003), estudia las fuentes de las fluctuaciones económicas y sus consecuencias para la elección del régimen cambiario en los principales países de América Latina. Mediante un VAR, encuentra que los choques externos juegan un papel limitado en las fluctuaciones del producto en estos países. Sugiere además que la rigidez del tipo de cambio no puede ser tan costosa para estas economías, como predice la teoría económica convencional. La literatura para el caso de Ecuador es escasa. Uno de los mayores obstáculos para establecer las características claves del ciclo económico ecuatoriano es la ausencia de datos trimestrales de algunas variables macro que juegan un rol importante en la teo- 
ría moderna de los ciclos económicos. No obstante, en este trabajo se trata de describir la mayor cantidad de hechos que los datos lo permitan.

\section{Metodología y datos}

Los hechos estilizados que caracterizan el ciclo económico en Ecuador fueron obtenidos con base en los datos disponible con información trimestral, la cual cubre el período 1990-2009. Para caracterizar las regularidades del ciclo económico en Ecuador se utiliza la metodología utilizada por Kydland y Prescott (1990) como una guía. Estos autores utilizan el Filtro ${ }^{6}$ de Hodrick y Prescott para obtener el componente cíclico de las series de datos.

Durante los últimos años un gran debate se ha producido con respecto a las habilidades de los diferentes métodos estadísticos para descomponer las series de tiempo en términos de tendencia y ciclo (Baxter y King, 1995; Canova, 1994). Las relativas ventajas de diferentes técnicas tal como las de Beveridge y Nelson (1981), Watson (1886), Hodrick y Prescott (1990) y Baxter y King (1995) no han sido establecidas y es posible cuestionar la solidez de los resultados obtenidos dependiendo el uso de cualquiera de ellos.

Los filtros mecánicos han sido criticados por varios autores, en Harvey y Jaeger (1993), ellos señalan que el filtro Hodrick-Prescott (H-P) puede producir ciclos espurios cuando se aplica a un conjunto de datos. Guay y St-Amant (1996) encuentran que los filtros HP y Baxter-King (B-K) no son buenos en identificar el componente cíclico de las series de tiempo que tiene un espectro con la forma características de la mayoría de las series macroeconómicas. Baxter y King (1995) señalan que los dos filtros tanto el HP como el BK están mal definidos al principio y al final de la muestra.

Sin embargo, a pesar de que este debate todavía no está resuelto, en este trabajo se sigue la práctica estándar de la literatura de los ciclos económicos, de reportar los hechos estilizados usando la desviación de las variables desde su tendencia de largo plazo obtenida con el filtro Hodrick y Prescott (H-P). Al hacer esta elección se facilita la comparación entre los resultados obtenidos aquí

$6 \quad$ La descripción del Filtro Hodrick y Prescott se desarrolla en el Anexo II. 
y los otros obtenidos de diferentes trabajos. Canova (1994) señala su apoyo a la utilización del filtro HP, argumentando que para comparar resultados entre diferentes modelos se debe estar viendo a través de la misma ventana (looking through the same window).

El reporte de hechos estilizados sigue a la literatura tradicional al presentar estadísticas con relación a cuatro aspectos del comportamiento cíclico de las series. ${ }^{7}$

1. La amplitud de las fluctuaciones (volatilidad) medida por la desviación estándar de cada serie. La volatilidad, al estar las series expresadas en logaritmos, corresponde a la desviación estándar del porcentaje en el que el componente cíclico de la variable se desvía del componente de crecimiento.

2. La razón de la desviación estándar de cada serie y el producto (volatilidad relativa).

3. El grado de persistencia o inercia en las desviaciones cíclicas medidas por el coeficiente de correlación de primer orden.

4. El grado y dirección de movimiento con el producto real, representado por la correlación contemporánea.

5. La fase de cambio o desfase con que una variable sigue a la otra, representado por la correlación rezagada entre ambas series (lidera o rezaga el ciclo del producto). ${ }^{8}$ Una variable lidera al producto por $i$ trimestres si su correlación llega a su valor más alto $i$ trimestres antes que el producto. Dado que todas las variables están en logaritmos, el cambio en el componente de tendencia presenta la tasa de crecimiento.

Adoptando la estructura de estudios anteriores se organiza la presentación de los hechos estilizados del ciclo económico siguiendo la siguiente clasificación de los datos: datos de insumo de producción, datos de los componentes de la demanda, datos de variables nominales. También se reportan las propiedades

7 Todas las series son ajustadas estacionalmente usando el procedimiento X-11 y expresadas de forma logarítmica antes de ser filtradas, con la excepción de las variables porcentuales, tales como la inflación y la tasa de interés las cuales van en niveles.

8 Si la correlación contemporánea es cercana a uno se describe a la variable como procíclica, si ésta es cercana a menos uno, la designamos como contra-cíclica, y si es cercana a cero, decimos que es a-cíclica. 
de ciertas variables relevantes para Ecuador al ser una economía pequeña y abierta.

\section{Evidencia empírica}

El gráfico 2 indica la evolución del componente cíclico del PIB de Ecuador en el período bajo análisis. En la serie filtrada, usando el algoritmo de BryBoschan ${ }^{9}$ para determinar las simas y los valles, se identifican cuatro diferentes ciclos (medido de cima a cima), sin embargo, ellos difieren en magnitud y tamaño. ${ }^{10}$ Cabe notar, que la amplitud de los ciclos ha disminuido en el tiempo, al igual que la volatilidad.

Gráfico 2. Componente cíclico del PIB real: 1990:1-2009:4

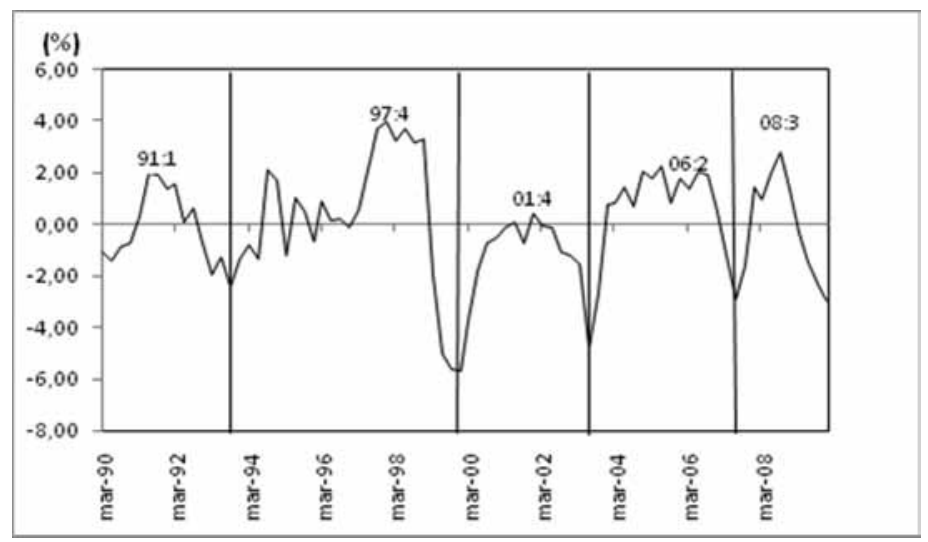

Fuente: Banco Central del Ecuador Elaboración Propia

9 La descripción de esta metodología se encuentra desarrollada en el Anexo II.

10 Se denomina amplitud a la diferencia entre el valor máximo y mínimo del ciclo y tamaño a la duración trimestral en cada fase del ciclo. 
La duración promedio de un ciclo durante este período es aproximadamente de cuatro años. Claramente la duración en la fase de recesión ha ido disminuyendo y son menores que las fases de expansión. La recuperación en la fase de expansión es altamente volátil en Ecuador, aproximadamente cinco trimestres. La mayor volatilidad del PIB se alcanzó en el período comprendido entre 1997: 4-2001: 4 alrededor de 3,24\%, los otros ciclos registran una volatilidad promedio de $1,90 \%$.

\section{I El PIB y los componentes de la demanda agregada ${ }^{11}$}

La primera característica notable, que se sitúa como un hecho importante es la alta volatilidad del PIB cercana a 2,09\% durante todo el período de análisis. Comparando con otros países de América Latina, Restrepo y Reyes (2000) reportan para Colombia una volatilidad de 1,61\%. Para Argentina Kydland y Zarazaga (1997) documentan una volatilidad de las fluctuaciones del producto dos veces mayor que las del Ecuador. Sin embargo, estas volatilidades son mayores a las reportadas para economías industrializadas. ${ }^{12}$ En parte esta alta volatilidad es un reflejo de las características estructurales de la economía ecuatoriana (relativa ausencia de estabilizadores automáticos, mercados financieros poco desarrollados, poca diversificación de la estructura productiva). Otra característica notable es la alta persistencia (autocorrelación) del PIB, un coeficiente de 0,77; es decir cuando el producto cae (sube) esta caída (alza) tiende a perdurar y a ser más pronunciada.

No obstante, a pesar de que la volatilidad del producto después de la dolarización es menor, esta diferencia no es estadísticamente significativa. Por lo tanto, este resultado indica que la volatilidad del producto es igual en ambos períodos. Este hecho es importante ya que trabajos como Edwards (2005) y Duncan (2003) encuentran que la volatilidad del producto es una función decreciente del régimen cambiario. A mayor flexibilidad del tipo de cambio menor la

11 Los cuadros, de esta sección, con los principales momentos de cada variable se encuentran en el Anexo III.

12 Volatilidad en Europa fue de 1,01\% en el período 1970-1990. Para los EE.UU. Kydland and Prescott encuentran una volatilidad de $1,71 \%$. 
volatilidad del producto. Así la ausencia de política monetaria y de tipo de cambio podría inducir a mayor volatilidad del producto. Sin embargo, la habilidad de la autoridad monetaria para acomodar los shocks reales va a depender de su grado de credibilidad y de su capacidad para distinguir en forma relativamente rápida entre shocks permanentes y transitorios.

En la ausencia de uno de ellos, la acción de la autoridad monetaria pasaría a ser desestabilizadora, produciendo mayores costos en términos de inflación. Este hecho fue notable en la crisis de 1999, las intervenciones del gobierno provocaban mayor inflación y no lograban estabilizar el producto, la carencia de credibilidad de la autoridad monetaria se debió en parte a la falta de independencia del Banco Central y, por otro lado, al historial de mal manejo de la política monetaria. No obstante, la evaluación de este tipo de opciones de política necesita mayor análisis empírico.

El consumo privado es altamente procíclico como se aprecia en la mayoría de los países, además se mueve en forma contemporánea con el producto. Cuando se mira todo el período analizado se encuentra que el consumo es más volátil ${ }^{13}$ que el producto. Siguiendo los modelos RBC que son esencialmente neoclásicos, el consumo es usualmente modelado bajo la hipótesis del ingreso permanente, en este sentido la volatilidad de consumo debería ser más pequeña que la del producto dado que los agentes que optimizan intertemporalmente tienden a suavizar el consumo (De Gregorio, 2007).

Sin embargo al realizar un análisis del ciclo del consumo se encuentra un hecho importante, este aparente exceso de variabilidad se debe principalmente a la gran volatilidad del consumo que se reporta para el período 1999-2000. Estadísticamente la volatilidad del consumo es diferente y muy superior a las volatilidades observadas en los otros períodos que se resumen en el Cuadro 1. Es importante destacar que al no incluir en el período comprendido de 19902009 la volatilidad observada en la época de crisis (1999-2000), la volatilidad

13 Este aparente exceso de volatilidad del consumo puede ser en parte, el resultado de usar los datos de consumo total. Así en general el consumo de durables es mucho más volátil que el consumo de no durables (De Gregorio (2007)). Sin embargo en Ecuador no se dispone de cifras donde se desagregue cada tipo de consumo como si ocurre en EE.UU. 
del consumo es estadísticamente igual a la del PIB, esta alta volatilidad sugiere que las restricciones al acceso al crédito en Ecuador son relevantes.

Cuadro 1. Volatilidad del ciclo del consumo

\begin{tabular}{lcc}
\multicolumn{1}{c}{ Período } & Absoluta & Relativa* $^{*}$ \\
\hline 1990-2009 & $2,33 \%$ & 1,11 \\
\hline 1990-2009** & $1,72 \%$ & 0,97 \\
\hline $1999-2000 * * *$ & $3,27 \%$ & 1,52 \\
\hline $1990-1998$ & $1,80 \%$ & 1,02 \\
\hline $2001-2009$ & $1,63 \%$ & 0,91 \\
\hline${ }^{*}$ En relación al PIB & \\
${ }^{* *}$ No incluye el período 1999-2000 \\
${ }^{* * *}$ Significativamente diferente al 1\%
\end{tabular}

El comportamiento del consumo en el período 1999-2000 fue distinto. En anteriores recesiones sufridas en Ecuador, el consumo disminuía en alrededor del 3\%, pero a mediados de 1999 el consumo sufrió una contracción cercana al $7 \%$ de su nivel de largo plazo.

Cuadro 2. Principales indicadores económicos

\begin{tabular}{|l|c|c|c|}
\hline & 1998 & \multicolumn{1}{c}{1999} & 2000 \\
\hline Producto interno bruto & 0.4 & $-6,7$ & 2 \\
\hline Inflación & 43.4 & 60,7 & 96,6 \\
\hline Salario mínimo real & -7.2 & $-10,7$ & -4 \\
\hline Dinero(M1) & 34.8 & 88,6 & $\ldots$ \\
\hline Tipo de cambio real efectivo & 3.8 & 38,7 & 18,1 \\
\hline Términos de Intercambio & -11 & 6,7 & 14,3 \\
\hline & & & Porcentajes \\
\hline Tasa de desempleo urbano & 11.5 & 15,1 & 14,7 \\
\hline Resultado fiscal/PIB & -5.6 & $-4,6$ & 0 \\
\hline Tasa de interés real pasiva & 2.6 & -2 & -43 \\
\hline Tasa de interés real activa & 10.3 & 7,9 & 38,6 \\
\hline Deuda externa /PIB & 65 & 97 & 100 \\
\hline
\end{tabular}




\begin{tabular}{l|c|c|c|} 
& & \multicolumn{2}{c}{ Millones de dólares } \\
\hline Exportaciones & 5.007 & 5.264 & 5.845 \\
\hline Importaciones & 6.409 & 3.761 & 4.165 \\
\hline Saldo cuenta corriente & -2.169 & 956 & 750 \\
\hline Cuenta de capital y finan- & 1.335 & -1.847 & -950 \\
\hline ciera & -834 & -891 & -200 \\
\hline
\end{tabular}

Fuente: Banco Central del Ecuador Elaboración Propia

En 1998, Ecuador sufre una fuerte caída de los términos de intercambio, debido a la disminución de los precios del petróleo, además de una grave destrucción de los cultivos causadas por un fenómeno climático. Estos dos shocks provocaron un déficit de cuenta corriente superior al $8 \%$ del PIB. La economía necesitaba financiar este déficit mediante un aumento de su deuda, o una diminución de sus reservas existentes de activos externos como lo hacía en sus anteriores recesiones, sin embargo la crisis financiera que se desató en 1999 trajo como consecuencia el congelamiento de las cuentas bancarias del sistema financiero local, una fuerte deuda interna, disminución de las reservas internacionales para controlar la devaluación de la moneda, además de restricciones internacionales de crédito, por lo tanto para poder ajustar el déficit los agentes tenían que reducir fuertemente su consumo interno.

En toda la muestra el consumo del gobierno está positivamente correlacionado con el PIB. El consumo del gobierno es procíclico y coincide con el ciclo del PIB. Este resultado difiere de la evidencia encontrada para los países industrializados, donde el comportamiento es a-cíclico. La alta volatilidad de esta variable sugiere que el consumo del gobierno podría jugar un rol importante en causar fluctuaciones económicas.

Otra característica relevante es la menor volatilidad de esta variable después de la dolarización. Savastano (1999) argumenta que uno de los beneficios de la dolarización oficial es que genera disciplina fiscal ya que elimina la posibilidad de la emisión monetaria para financiar el déficit fiscal. Otra característica interesante del ciclo económico en Ecuador es la presencia de grandes fluctuaciones en la inversión. Como fracción del PIB, la formación bruta de 
capital fijo aumentó desde un bajo $17 \%$ a principios de 1990 a un $28 \%$ a finales de 2009. Cabe decir que esta importante expansión de la inversión fue también caracterizada por altos niveles de volatilidad, que alcanzaron un $7,96 \%$ en el período de estudio, esto es cuatro veces más que el PIB. Estos resultados son consistentes con la evidencia internacional, Kydland y Prescott para los EE. UU.; Fisher, et al. para Australia; Kydland y Zarazaga para Argentina.

Las exportaciones e importaciones son respectivamente dos y cuatro veces más volátiles que el PIB en la muestra completa. Para los EE.UU. Kydland y Prescott reportan que las importaciones y las exportaciones son tres veces más volátiles que el PIB. La mayor volatilidad relativa de las exportaciones con respecto al PIB se debe a que las exportaciones representan cerca del $35 \%$ del PIB, mientras tanto para los EE.UU. representan solo un 13\%. Las importaciones son altamente procíclicas, no obstante las exportaciones son débilmente procíclicas. El patrón procíclico de las importaciones puede estar asociado a la fuerte prociclicidad de la inversión. Un resultado interesante surge de la menor volatilidad del componente cíclico de las importaciones después de la dolarización, esto puede ser una consecuencia directa de la menor volatilidad del tipo de cambio, ya que con la dolarización se reducen los costos de transacción relacionados al comercio de bienes.

Gráfico 2. Comportamiento cíclico del PIB y las exportaciones

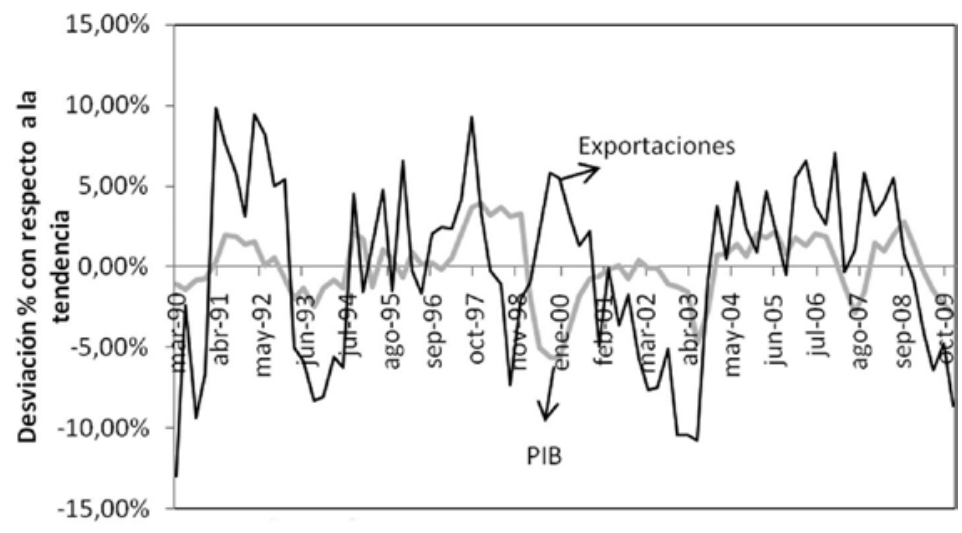

Fuente: Banco Central del Ecuador Elaboración Propia 
Un hecho importante es que el componente cíclico de las exportaciones lidera al ciclo del PIB por cerca de dos trimestres, este comportamiento es común en toda la muestra, lo que resalta el importante rol que juegan las exportaciones en las fluctuaciones de corto plazo en una economía pequeña y abierta como la ecuatoriana. Dado que la composición de las exportaciones no ha cambiado en los dos períodos y al ser estos principalmente commodities (petróleo y banano), la volatilidad no ha cambiado en los dos sub períodos.

\section{V.2 Factores productivos}

En la literatura de ciclos reales, estimulada por Kydland y Prescott (1982), el mercado laboral es fundamental para la transmisión de los shocks productivos en la economía. Este mercado concentra, además algunos de los desafíos más relevantes que enfrenta esta literatura. ${ }^{14}$ Los mercados de factores, por medio de políticas gubernamentales, contienen rigideces que los alejan del equilibrio obtenido en modelos sin distorsiones. Las rigideces de los mercados de factores son evidentes en Ecuador. Así el stock de capital muestra un comportamiento acíclico para toda la muestra. Este tiende a rezagar al ciclo del producto por casi dos trimestres. Es decir, al stock de capital le toma dos trimestres ajustarse al ciclo económico.

Un hecho importante es el comportamiento altamente volátil de esta serie: 1,28 veces más volátil que el PIB, este resultado es contradictorio a la evidencia internacional, los mismos reportan que el stock de capital fluctúa mucho menos que el producto. Sin embargo, es importante tomar en cuenta que la volatilidad del capital respecto al PIB está determinada por sus componentes. Restrepo y Soto (2004) encuentran para Chile que en el caso de la maquinaria, la volatilidad del capital es más alta que la del producto, pero ésta se ve contrarrestada por la baja variabilidad que presenta el capital de construcción. No obstante Ecuador no cuenta con tal desagregación del stock de capital. Otro hecho importante lo reporta el salario real, es altamente volátil, nueve veces más que

14 La mayoría de las extensiones realizadas a la teoría de los ciclos reales durante los últimos años tiene relación con el mercado laboral. Por ejemplo, incorporando indivisibilidades (Hansen, 1985) y asociando el ocio con trabajo en casa (Greenwood et al., 1995). 
el PIB; según la teoría Keynesiana se esperaría una conducta contra-cíclica de dicha variable y de acuerdo, a los modelos de equilibrio del ciclo económico se predeciría un comportamiento procíclico. En Ecuador el salario real tiene un comportamiento procíclico y rezaga al ciclo del producto por dos trimestres. Esta característica es común en los países en desarrollo, mientras que en los países desarrollados son más bien acíclicos. Esta evidencia apuntaría a que la curva de Phillips basada en rigideces de los salarios nominales no es realista, pues este tipo de modelos predice un salario real contra-cíclico (De Gregorio, 2007).

En los modelos de ciclo económico real, la incorporación de sustitución intertemporal del trabajo, permite generar una correlación positiva entre el empleo y el producto, este hecho también se observa en la serie cíclica del empleo, ésta presenta un comportamiento pró-cíclico y es menos volátil que el PIB, hecho que también se reporta para economías desarrolladas y emergentes.

\section{V.3 Agregados monetarios, tasa de interés y precios}

El comportamiento de las variables nominales tradicionalmente ha sido sujeto de mucha atención en las investigaciones del ciclo económico. Para analizar el comportamiento cíclico de los agregados monetarios que incluyen $M 1$ y $M 2$, es importante separar el análisis pre y post dolarización, ya que la dolarización restringe la emisión monetaria y la capacidad de la autoridad de utilizar política monetaria por lo tanto la oferta monetaria será el resultado de la balanza de pagos (Rojas-Suárez, 1999).

Así, en el período 1994-1999, M1 es seis veces más volátil que el producto y refleja el interés de la política monetaria seguida por el Banco Central del Ecuador. M1 lidera al ciclo hasta por tres trimestres al ciclo del producto. La correlación contemporánea entre el PIB y M2 es cero. No obstante existe una correlación positiva que lidera al producto. Por lo tanto el mito monetario que encuentra Kydland y Prescott en los EE.UU. ya que M1 coincide con el ciclo del PIB, parece ser una realidad en Ecuador antes de 2000. Mientras que el 'cuasidinero' (M2-M1) tiene un comportamiento acíclico, no obstante esta lidera al ciclo del PIB. 
Después de la dolarización el comportamiento de M1 es acíclico, no obstante M2 es procíclico y rezaga al ciclo por solo un trimestre, por lo tanto, como señala Kydland y Prescott el análisis del mercado del crédito puede ocupar un lugar importante en el estudio del ciclo económico ecuatoriano antes y después de la dolarización. Un hecho semejante en las dos submuestras es la alta volatilidad de M1. Sin embargo, la volatilidad de M1 en el período predolarización es dos veces menor que la encontrada en el período posdolarización.Los resultados son consistentes con los encontrados en la literatura internacional (Backus and Kehoe, 1992).

Por otro lado, al analizar el comportamiento del ciclo de la tasa de interés se encuentran diferencias notables en las dos submuestras. Así, en la primerasubmuestra las tasas de interés de corto plazo nominales, muestran un patrón procíclico y coincidente con el ciclo del producto, lo cual indicaría una política monetaria más activa. Por lo tanto, aumentos en el producto estarían seguidos por aumentos en la tasa de interés de corto plazo, indicando una política acomodativa. Por el contrario, en el período de adopción de la dolarización, la tasa de interés de corto plazo muestra un patrón altamente anticiclico, esto debido a que en una economía dolarizada la evolución de la tasa de interés de corto plazo se ve influenciada por la tasa de interés internacional, a través de la condición de paridad de tasas de interés, y a través del premio por riesgo, el comportamiento de la tasa de interés va a depender del grado de sincronización del ciclo de los EE.UU. con el de Ecuador (Carrera et al., 2002) y de la proporción de premio por riesgo.

La tasa de interés en todo el período es altamente volátil, no obstante después de la dolarización ésta es cuatro veces menor que la volatilidad reportada para el período 1990-1999.

En la literatura de ciclos económicos, las teorías de oferta agregada y ciclo económico real establecen que si los movimientos del producto se producen debido a shocks de demanda, se espera que los precios sean procíclicos con el producto, por el contrario, si los shocks se originan por el lado de la oferta luego se espera que los precios sean contracíclicos. En toda la muestra de estudio, los precios indican un comportamiento contracíclico con el producto. La importancia de estos hechos es que los shocks relacionados a cambios tecnológicos, cambio en los precios o cambio en los términos de intercambio, podrían ser los 
responsables de los ciclos económicos en Ecuador. Otro resultado interesante es que los precios son cuatro veces más volátiles que el PIB, confirmando el comportamiento inflacionario del país, además los precios muestran una alta inercia inflacionaria, el coeficiente de autocorrelación es de 0,94 . Este comportamiento es similar en las dos submuestras.

Al examinar el comportamiento de la inflación, se resalta nuevamente el carácter contra-cíclico de dicha variable, similar a los precios, estos dos resultados respaldan el hecho que los salarios reales sean contra-cíclicos, sugiriendo que los shocks de oferta son una importante fuente de fluctuaciones en la actividad agregada. Otro hecho importante que se debe destacar para el período 1990-1999, es el comportamiento cíclico del tipo de cambio nominal, el cual a su vez es contra-cíclico y rezaga al ciclo del PIB por un trimestre, reafirmando la evidencia reportada anteriormente: en épocas de recesión la autoridad monetaria devaluaba la moneda, como un mecanismo para atenuar el ciclo del PIB. Sin embargo las depreciaciones cambiarias pueden afectar directamente el precio de los bienes de producción nacional si la política monetaria carece de credibilidad (De Gregorio, 2009). Asimismo los agentes probablemente reaccionarán frente a la depreciación nominal subiendo el precio de los bienes nacionales. En estrecha relación, si las expectativas de inflación no están bien ancladas, los aumentos iniciales de los precios de algunos productos importados se pueden propagar a otros precios internos y producir alzas generalizadas en los precios. En tal situación habrá un alto pass-through a la inflación del IPC.

En el caso de la economía ecuatoriana para el período 1990-1999, no existe ningún tipo de estudio realizado anteriormente relacionando este tema. Al realizar un simple análisis de correlación entre precios internos y el tipo de cambio, se encontró que existe una alta correlación positiva cercana a 0,80 y que los precios internos rezagan al tipo de cambio un trimestre. Para poder confirmar el resultado anterior, se calculó el coeficiente de pass-through, ${ }^{15}$ el cual resultó estar cercano a 0,70 en promedio. A primera vista, esto podría ayudar a corroborar el hecho de que la falta de credibilidadde la autoridad monetaria hizo que

15 El coeficiente de pass-throught es el coeficiente de la depreciación cambiaria anual tomada de una regresión para la inflación anual, sin otros regresores utilizando datos mensuales y una regresión con muestra móvil a diez años. Metodología tomada de De Gregorio (2009). 
las expectativas de inflación no estuvieran bien ancladas, dando como resultado que las variaciones en el tipo de cambio nominal produjeran un fuerte aumento en los precios.

Sin embargo, al ser sólo un pequeño análisis no podemos obtener conclusiones robustas al respecto, por lo cual es un hecho importante que merece mayor atención para la aplicación de futuras investigaciones.

\section{V.4 Aspectos Internacionales del Ciclo}

Finalmente, en una economía pequeña y abierta, tal como la ecuatoriana, se debería esperar un importante efecto del ciclo económico internacional en la economía doméstica. Principalmente cuando el comercio exterior es fuertemente dependiente de los precios de los commodity. En este caso, esta dependencia surge de la gran proporción de petróleo en las exportaciones. Dado que los precios de los commodity son altamente volátiles por tal motivo, no es sorprendente encontrar que la volatilidad de los términos de intercambio sea cinco veces más volátil que el PIB. Esta volatilidad es mucho más grande que las reportadas para economías desarrolladas, las mismas que en promedio presentan una volatilidad de 3,5\%. No obstante, según Mendoza (1995), reporta una volatilidad en promedio de alrededor de 12\% para economías de América Latina y África.

No existe diferencia significativa de la volatilidad de los términos de intercambio en los períodos antes y después de la dolarización, el patrón de comovimiento es similar en todo el período muestral, siendo este débilmente procíclico con el ciclo del PIB, a pesar de ello lidera al PIB por tres trimestres, evidenciando que los shocks de términos de intercambio son una de las principales fuentes que conducen al ciclo económico ecuatoriano.

Mendoza (1995) muestra evidencia para un conjunto de economías emergentes que los choques a los términos de intercambio son largos, persistentes y débilmente procíclicos. El estudio de Mendoza señala que los choques a los términos de intercambio explican entre un 40 y $50 \%$ de la variabilidad del producto. Este resultado ofrece indicios de que las desviaciones de los términos de intercambio al ser volátiles y persistentes, dado el movimiento procíclico anticipado con el producto, podrían conllevar a ciclos en el producto. El análisis 
del comportamiento del tipo de cambio real es de gran relevancia ya que esta variable juega un rol determinante al describir el grado de competitividad de la economía frente al resto del mundo (Larrain y Sachs, 2005).

En lo que se refiere al comportamiento cíclico del tipo de cambio en el período 1990-1999, el TCR claramente es más volátil, comprobando la hipótesis de Mussa (1986) que el tipo de cambio real es más volátil bajo un tipo de cambio flexible. Esto se debe principalmente por el comportamiento del tipo de cambio nominal. Además, en este período la volatilidad del tipo de cambio real está fuertemente influenciado por la variabilidad del tipo de cambio nominal. Sin un completo pass-through al IPC, los movimientos del tipo de cambio nominal se traducen en movimientos del tipo de cambio real. Esto afirma el comportamiento altamente contracíclico y coincidente del tipo de cambio real con el ciclo del PIB.

En el segundo subperíodo el TCR es estadísticamente menos volátil que el período anterior, debido a que con la adopción de la dolarización la autoridad monetaria ya no ejerce poder de uso sobre el tipo de cambio nominal. Sin embargo, el TCR tiene un comportamiento débilmente procíclico con el PIB y rezaga al ciclo por tres trimestres. Para entender por qué se presenta este comportamiento procíclico del TCR, se debe analizar los principales fundamentales que tienen influencia sobre el tipo de cambio real. Este tipo de análisis está fuera del alcance de estudio de la presente investigación.

Siguiendo el trabajo de Segovia ${ }^{16}$ (2003), el cual determinó las variables fundamentales que establecen la trayectoria del TCR en Ecuador, se aplicó la misma metodología para dos períodos: antes (1990-1999) y después (20002009) de la dolarización, para determinar la duración de los desalineamientos del TCR, mediante un modelo vectorial de corrección de errores. Los resultados obtenidos indicaron que los desalineamientos del tipo de cambio real duraron en promedio dos trimestres antes de la dolarización, mientras que después de ésta el período promedio fue de seis trimestres.

16 Estos autores realizan un estudio para el período 1994-2002, y encuentran que los fundamentales del tipo de cambio real para Ecuador son: Productividad media del trabajo(-), remesas(+), Flujo de capitales(-), exportaciones petroleras(+), términos de intercambio(-) y la apertura comercial(+). 
En consecuencia, después de la dolarización, cuando golpea un shock real a la economía ecuatoriana, el tipo de cambio real se ajusta más lentamente a su valor fundamental. Este mayor tiempo del desalineamiento del TCR podría provocar desajustes económicos. Está claro que estos resultados no reflejan evidencia concluyente, sino que pueden ser tomados como referencia para futuras investigaciones que analicen la importancia de los desalineamientos del TCR en la economía ecuatoriana y sus posibles implicancias de política económica.

\section{Resumen de resultados}

Este trabajo presenta un conjunto de "hechos estilizados" sobre las fluctuaciones cíclicas de la economía ecuatoriana, usando la metodología ateórica desarrollada por Kydland y Prescott (1990). Las propiedades de las fluctuaciones económicas se analizaron utilizando el filtro Hodrick-Prescott. Se documenta además el cambio generado sobre la volatilidad y los comovimientos de las principales variables macroeconómicas a partir de la adopción de la dolarización a inicios del año 2000. Los resultados obtenidos y futuras extensiones de este trabajo se resumen en los siguientes puntos:

En todo el período de análisis el comportamiento del consumo de los hogares es altamente procíclico, esta característica demuestra un exceso de sensibilidad a las fluctuaciones del producto, el mismo que revela las restricciones de crédito en la economía ecuatoriana.

Como se mencionó anteriormente, la inversión es muy volátil, es cuatro veces mayor que el PIB. El comportamiento de dicha variable es igual en los dos subperíodos analizados.

Las exportaciones muestran un patrón procíclico y son dos veces más volátiles que el PIB. Una característica importante, poco común en las economías desarrolladas, es el comovimiento anticipado de las exportaciones, este hecho podría revelar el rol protagónico de las exportaciones en la determinación de las fluctuaciones de corto plazo en una economía pequeña y abierta como la de Ecuador. Este resultado es confirmado por el comportamiento de los términos de intercambio el mismo que lidera al ciclo del PIB por tres trimestres. 
Otro hecho importante es la disminución de la volatilidad de las importaciones después de la adopción de la dolarización, esta característica podría ser producto de la conducta menos volátil del tipo de cambio nominal.

El comportamiento de los insumos productivos es constante en todo el período. El empleo es procíclico y menos volátil que el ciclo del PIB, una característica importante es el comportamiento del salario real que es procíclico. A pesar que la meta de este trabajo no es defender una teoría acerca de la naturaleza de los shocks que afectan a la economía, el comportamiento del salario real es un hecho importante a favor de los shocks de oferta.

Este resultado es reforzado por el comportamiento contracíclico de los precios y la inflación, sobre todo el período de análisis.

Las variables de política monetaria son altamente volátiles, su comportamiento cambia después de la adopción de la dolarización. En la etapa predolarización las fluctuaciones de M1 son fuertemente procíclicas y lideran al ciclo por un trimestre. Estos resultados refuerzan el papel protagónico del dinero, es decir el "mito monetario" reportado por Kydland y Prescott (1990). Para EE.UU. podría ser una realidad en Ecuador.

Sin embargo, después de la dolarización M1 pierde su papel protagónico como resultado del ancla nominal adoptada por Ecuador. No obstante M2 es procíclico y rezaga al ciclo por solo un trimestre, por lo tanto, como señala Kydland y Prescott el análisis del mercado del crédito puede ocupar un lugar importante en el estudio del ciclo económico ecuatoriano.

Otro que resulta importante es el comportamiento del tipo de cambio real, cuya volatilidad disminuye considerablemente después de la adopción de la dolarización. Mas al realizar un pequeño análisis se reporta que el TCR aumentó la duración del desalineamiento después de la dolarización. 


\section{Bibliografía}

Agenor, P., J. McDermontt y E. Prasad. "Macroeconomic fluctuations in developing countries: some stylized facts". The World Bank Economic Review 14 (2): 251-85. 1998.

Ahmed, S. "Sources of economic fluctuations in Latin America and Implications for choice of exchange rate regimes". Journal of Development Economics 72: 181: 202. 2003.

Backus, D. y P. Kehoe. "Internacional evidence on the Historical Properties of Business Cycles". American Economic Review, septiembre, 1982. 1992.

Baxter, M. "Business cycles, stylized facts, and the exchange rate regime: evidence from the United States". Journal of Internacional Money and Finance N. ${ }^{\circ}$ 10: 71-88. (1991).

Baxter, M y R. King. "Measuring Business Cycles: Approximate Band-Pass Filters for Economic Time Series". National Bureau of Economic Research, Working Paper N. ${ }^{\circ}$ 5022. 1995.

Belaisch, A. y C. Soto. "Empirical Regularities of Chilean Business Cycles". Banco Central de Chile, Documento de Trabajo N. ${ }^{\circ} 41.1998$.

Berg, A. y E. Borensztein. "Full Dollarization: The Pros and Cons". IMF Policy Discussion Paper. Washington DC. 2000.

Berg, A., Borensztein, E. y P. Mauro. "An Evaluation of Monetary Regime Options for Latin America". Central Bank of Chile. Working paper N. ${ }^{\circ} 178$. (2002).

Beveridge, S. y R. Nelson. "A New Approach to Decomposition of Economic Time Series into Permanent and Transitory Components with Particular Attention to Measurement of the "Business Cycle". Journal of Monetary Economics, vol. 7, pp. 151-174. 1981.

Bradbury, M. y M. Vernengo. "The Limits to Dollarization in Ecuador: Lessons from Argentina". University of Utah. Working paper N. ${ }^{\circ}$ 2008-12. 2008.

Broda, C. "Terms of Trade and Exchange Rate Regimes in Developing Countries". Federal Reserve Bank of New York. 2002. 
Burns, A. y W. Mitchell. "Measuring Business Cycle”, New York: National Bureau of Economic Research. 1941.

Canova, F. "Detrending and business clycles facts". Journal of Monetary Economics 41: 475-512. 1994.

Calvo, G. “On Dollarization”. University of Maryland, Economics of transition April 20. 1999.

Cashing, P. y S. Ouliaris. "Key Features of Australian Business Cycles". University of South Australia. 2004.

Chang, R. "Dollarization: A scorecard". Federal Reserve Bank of Atlanta. Economic Review, Third Quarter. 2000.

Cortés, H. "Lecciones del Pasado Recesiones Económicas en Chile: 19261982”, Cuadernos de Economía N. ${ }^{\circ}$ 63, pp. 137-168. 1984.

De Gregorio, J. Macroeconomía: Teoría y política. 1era. Edición. Prentice Hall. 2007.

Duncan, R. "Exploring the implications of official Dollarization on Macroecnomic Volatility", Central Bank of Chile, Working Paper N. 200. $2003 a$.

Duncan, R. "Floating, Official Dollarization, and Macroeconomic Volatility: an Analysis for the chilean Economy”. Central Bank of Chile, Working Paper N. ${ }^{\circ}$ 249. 2003 b.

Edwards, S. y I. Magendzo. "Dollarization, Inflation and Growth". NBER Working Paper 8671, December. 2001.

Fisher, L., Otto, G. y G. Voss. “Australian Business Cycles Facts”. University of New South Wales. 1994.

Franken, H., Le Fort, G. y E. Parrado. "Business Cycle Dynamics and Shocks resilience in Chile”. Central Bank of Chile, Working Papers N. 3 331. 2005.

Friedman, M. "The Case for Flexible Exchange Rates", Essays in Positive Economics, Universidad de Chicago. 1953.

Giannone, D. y L. Reichlin. "Trends and Cycles in the Euro Area. How Much Heterogeneity and Should We Worry About it". European Central Bank. Working paper N. ${ }^{\text {595. }} 2006$. 
Guay, A. y P. St-Amant. "Do Mechanical Filters Provide a Good Approximation of Business Cycles?”, working paper, Bank of Canada. 1996.

Hachette de la F, D., Ossa, F. y F. Rosende. "Aspectos monetarios y macroeconómicos de la integración”. Cuadernos de Economía N. ${ }^{\circ}$ 98: 153183. 1996.

Harvey, A. y A. Jaeger. "Detrending, Sytilized Facts, and the Business Cycle. "Journal of Applied Econometrics 8(3): 231-47. 1993.

Hodrick, R. y E. Prescott. "Postwar U.S. Business Cycles: an Empirical Investigation. Pitssburgh: Carnegie.-Mellon University; Discussion Papers 451, Northwestern University. 1980.

Hoffmaister, A. y J. Roldós. "The sources of macroeconomic fluctuations in developing countries: Brazil and Korea". Journal of Macroeconomics 23 (1). 2001.

Koopmans, T. "Measurement without theory". Review of Economic Statistics, Cowles foundation Paper 25a. (1945).

King, R.G., y C. I. Plosser. "Real Business Cycles and the Test of the Adelmans", Journal of Monetary Economics, N. * 33, pp. 405 - 438. 1994.

Kydland, F y E. Prescott. "Business Cycles: Real Facts and Monetary Myth", Federal Reserve Bank of Minneapolis, Quarterly Review. 1990.

Kydland, F y E. Prescott. "Time to Build and Aggregate Fluctuations". Econométrica 50 (November): 1345-70. 1982.

Kydland, F y E. Zarazaga. "Is the Business Cycle of Argentina Different?". Federal Reserve Bank of Dallas. Economic review fourth quarter. 1997.

Larraín, F., Tavares, J. y C. García. "Regional Currencies versus Dollarization: Options for Asia and the Americas". NBER. 2003.

Larraín, F. "Flotar o Dolarizar ¿Qué nos dice la evidencia?”. El trimestre económico vol. LXXII (1): 5: 28. 2005.

Larraín, F. y F. Parro. "Chile menos volátil”. El trimestre económico, vol. LXXV (3): 563-596. 2008.

Lucas, R. "Understanding business cycles", Carnegie Rochester Conference Series on Public Policy 5: 7-29. 1977. 
Mendoza, E. "The Benefits of Dollarization When Stabilization Policy Lacks Credibility and Financial Markets are Imperfect". Forthcoming, Journal of Money, Credit, and Banking. Agosto. 2000.

McKinnon, Ronald I., 'Optimun currency areas', The American Economic Review vol. 53, 717-125. 1963.

Raddatz, C.”Are external shocks responsible for the instabililty of output in low income countires?". Journal of Development Economics 84: 155-187. 2007.

Restrepo, J. y J. Reyes. "Los ciclos económicos en Colombia. Evidencia empírica (1977-1998)". Archivos de macroeconomía, documento N. ${ }^{\circ} 131$. 2000.

Rojas-Suárez, L. "Dollarization in Latin America?". Prepared Testimony. Prepared Testimony presented in Hearing on Official Dollarization in Latin America. U.S. Senate Banking Committee. 1999.

Savastano, M. Presentation prepared for the conference "Dolarizar la Economía Peruana: Riesgos y Oportunidades". Lima 1999.

Segovia, S. "Tipo de cambio real de equilibrio. Un análisis del caso ecuatoriano". Banco Central del Ecuador, Nota Técnica N. ${ }^{\circ}$ 71. 2003.

Schmitt-Grohé, S. and M. Uribe. "Stabilization Policy and the Costs of Dollarization". Journal of Money, Credit, and Banking, vol. 2001.

Envío 18 de septiembre/2011 - Aceptación 17 de noviembre/2011 